\title{
SOME RELATIONS BETWEEN WOLF-RAYET AND \\ P CYGNI-TYPE STARS
}

\author{
MART DE GROOT \\ European Southern Observatory, Casilla 16317, Correo 9, Santiago, Chile
}

\section{Introduction}

In taking up the subject of the relation between different types of early type stars one inevitably comes on ground set foot upon before by C. S. Beals. Already in $\mathbf{1 9 4 0}$ he published an extensive paper entitled 'On the Physical Characteristics of the WolfRayet Stars and Their Relation to Other Objects of Early Type' (Beals, 1940). The purpose of the present contribution is mainly to make the discussion more complete by including observations of later years.

One of Beals' biggest problems in 1940 was the lack of sufficiently detailed observations on the subject. Now, more than $\mathbf{3 0}$ years later, the situation has improved only a little. Hence, in discussing the characteristics of the P Cygni-type stars, one often can only refer to observations of P Cygni itself, because this is the only well-studied star among the P Cygni-type stars. However, it should be remembered that P Cygni, although the denominator of the group, probably is not really representative of what normally is called the group of P Cygni-type stars. This is because P Cygni has a rather early spectral type when compared to the other P Cygni-type stars and because none of the other stars in the group shows the P Cygni phenomenon as completely as P Cygni itself.

Beals (1940) remarked that "it is very difficult to set up any arbitrary division line between P Cygni and Be stars". However, this paper does not include a discussion of Be stars proper, the separation being made on the ground of the difference in spectrum. Be stars are all high-rotational-velocity stars, while P Cygni-type stars normally are normal-rotational-velocity supergiant-like stars. Beals (1940) also pointed out the close relationship between the $\alpha$ Cygni-type stars and the P Cygni-type stars; this close relation is retained in the present discussion.

\section{Definition}

The fundamental work of P Cygni-type stars is Beals' study (1950) in which he discusses the spectra of 69 stars showing in at least one line of their spectrum one (or more) of four typical spectral-line profiles. All these profiles have in common a nearly undisplaced emission component, flanked on its short-wave-length side by an absorption component. Beals' group of P Cygni-type stars is in fact a very heterogeneous group, ranging from spectral type O5feq to F2e $\alpha$. From his 69 stars 15 are $\alpha$ Cygni type (i.e. supergiants, normally showing only a P Cygni-type line profile in $H \alpha$ ), 3 are 
Of stars, 7 are close binaries of $\beta$ Lyrae type, 3 are symbiotic objects, one is a planetary nebula of low surface brightness and there are several unique stars ( $\eta$ Car, XX Oph).

For a discussion of the similarities of P Cygni-type stars and Wolf-Rayet stars one should try to limit the class of P Cygni-type stars to a more homogeneous group of stars. Since, in the context of WR stars it seems worthwhile to distinguish between WR objects ,quasi-WR objects and the WR phenomenon (Thomas, 1968), I suggest that we define similar terms for P Cygni-type stars, although on a different basis as Thomas' classification.

The P Cygni phenomenon, then, consists of the fact that there are stars showing in their spectra one or more of the four P Cygni-type line profiles defined by Beals (1950). Such stars will be called quasi-P Cygni-type stars if the number of their spectral lines showing the P Cygni phenomenon is very limited, e.g. if it is only shown in one spectrum, or, as even more often is the case, only in the first few Balmer lines. A pure P Cygni-type star is then a star showing a spectrum in which the $\mathbf{P}$ Cygni phenomenon is shown by a substantial number of spectral lines of at least two different elements. Such stars will conveniently be called P Cygni-type stars. Most supergiants of early spectral type are showing the P Cygni phenomenon in a rather limited sense (often only in $H \alpha$ ) and so present only an extreme case of the occurrence of the P Cygni phenomenon. They will be referred to as $\alpha$ Cygni-type stars and are forming part of the group of quasi-P Cygni-type stars.

In this context the close binaries of $\beta$ Lyrae type showing the P Cygni phenomenon will not be discussed. In those stars the P Cygni phenomenon is produced by the duplicity of the star and the presence of a gaseous ring or envelope encompassing the two stars. This selection yields a group of 34 pure P Cygni type stars out of Beals' original 69. There are, however, some more pure P Cygni-type stars not included in Beals' list. Nevertheless, the pure P Cygni-type stars still form a rather small group including quite different objects. Furthermore, good observations have only been made of a relatively small number of these stars. Theoreticians concerned with WR stars often feel a trifle embarrassed because, owing to the limited number of stars, there are only few observations available to compare their theoretical results with. In the case of the P Cygni type stars the situation is even more difficult, and so is a comparison of these two groups of stars.

\section{Galactic Distribution}

Because of the just-mentioned circumstance little can be said about the galactic distribution of the P Cygni-type stars. It should be remarked, however, that all known P Cygni-type stars are lying close to the galactic equator. Of Beals' P Cygni-type stars, apart from those in the Large Magellanic Cloud, only one star is found at a distance of more than ten degrees from the galactic equator. Also, since the P Cygni-type stars are closely related to the $\mathrm{O}$ and B-type supergiants, they, like the classical WR stars, belong to Population I. 


\section{The Line Spectrum}

As has been statcd earlier (cf. Section 2) the line spectrum of a P Cygni-type star is characterized by spectral lines showing a nearly undisplaced emission component flanked on its short-wave-length side by a violet-displaced absorption component. Due to the heterogeneity of the group a general description of the main spectral characteristics of the $\mathbf{P}$ Cygni-type stars cannot easily be given. The spectral types of the pure $\mathrm{P}$ Cygni-type stars range from $\mathrm{O} 6$ to $\mathrm{A} 4$ and this reflects well how different one P Cygni-type star can be from any other.

The most prominent spectra normally are those of $\mathrm{H}$ and $\mathrm{He} \mathrm{I}$, except for the extreme spectral types. In the early A stars the role of $\mathrm{He} \mathrm{I}$ as a prominent spectrum is taken over by $\mathrm{Fe}$ II, and $\mathrm{Ti}$ II. In the earlier spectral types, besides $\mathrm{H}$ and $\mathrm{He} \mathrm{I}$, lines of $\mathrm{N}$ II, $\mathrm{N}$ III, $\mathrm{O}$ II and Fe III often are present as medium strong lines. Especially in the B-type stars the N II multiplet between $\lambda 4601 \AA$ and $\lambda 4643 \AA$ appears to be stronger than in normal stars of the same spectral type. Abundance analyses along classical ways have been done for P Cygni itself by Ghobros (1962) and by Luud (1967a, b). The latter finds that the composition is essentially the same as for the early B-type supergiants with $\mathrm{N}$ probably a bit overabundant in $\mathrm{P}$ Cygni.

An abundance analysis by Caputo and Viotti (1970) of the spectrum-variable star AG Car suggests that this star, which has shown a variable number of P Cygni-type lines since 1950 (Thackeray, 1950, 1956; Bond and Landolt, 1970), is underabundant in $\mathrm{C}$ and $\mathrm{O}$.

Absorption-line radial velocities in P Cygni-type stars are found to vary with the excitation potential of the spectral lines from which they are determined. The normal interpretation is that this is produced by a stratified atmosphere showing a velocity gradient. Hutchings $(1968 \mathrm{a}, 1968 \mathrm{~b}, 1969)$ studied this effect for several stars and gave a method of determining the gradient of the excitation temperature in the atmosphere. Similar procedures have been applied by other authors and the general conclusion is that the radial velocities increase with distance from the stellar surface from a few tens of $\mathrm{km} \mathrm{s}^{-1}$ to a few hundreds of $\mathrm{km} \mathrm{s}^{-1}$ at 3 to 10 stellar radii. Rocket observations, e.g. by Morton (1967), have even indicated velocities up to $2000 \mathrm{~km} \mathrm{~s}^{-1}$ in the far-UV resonance lines of abundant elements such as $\mathrm{C}, \mathrm{N}$ and $\mathrm{Si}$.

The excitation temperature is highest near the stellar surface and decreases when going outward. This agrees with what Bappu said yesterday about the behaviour of the excitation temperature in WR stars.

It may be useful to compare some of Conti's information of this morning with observations of P Cygni. The latter star (spectral type B 1.5 according to the absorption lines) is showing the $C_{\text {III }} \lambda 5696$ line in emission and very weak absorption, but there is emission at $\mathrm{N}_{\text {III }} \lambda \lambda 4534-4640$. The unidentified lines at $\lambda 4485$ and $\lambda 4503$ found in Of stars and which seem to behave like C III are not found in P Cygni. The He I emission lines only found in the most luminous late-type Of stars all show P Cygnitype profiles in P Cygni. C III $\lambda 4650$ which only appears in emission in certain hot Of stars shows a very weak emission and absorption in P Cygni. In Of stars the same 
behaviour is found for the Si IV lines $\lambda 4088, \lambda 4116$; in P Cygni these lines only appear in absorption.

\subsection{INTERSTELLAR LINES}

The interstellar lines in the spectrum of P Cygni show a remarkable behaviour. Apart from a complex interstellar line with components between -10 and $-23 \mathrm{~km} \mathrm{~s}^{-1}$ there also are weaker components at $+17,0,-36$ and $-49 \mathrm{~km} \mathrm{~s}^{-1}$ (referred to the Sun) i.e. at $-33,-16,+19$ and $+33 \mathrm{~km} \mathrm{~s}^{-1}$ with respect to the star. Thus there occur weak interstellar lines at higher-than-normal velocities and in pairs suggesting both material moving away from and falling towards the star. Yesterday Bappu presented a similar observation in a WC8 star. No such behaviour of the interstellar lines is known for other P Cygni-type stars, but, again, this is largely due to a lack of high-dispersion spectrophotometric studies.

\section{The Continuous Spectrum}

The continuous spectrum of $20 \mathrm{P}$ Cygni-type stars has been studied by Arkhipova (1963). Although the stars studied do not form as homogeneous a group as one might wish it can be concluded that for pure P Cygni-type stars the colour temperature normally is lower than their excitation temperature. For three P Cygni-type stars the colour temperature varies inversely with wavelength to some appreciable extent in the sense that the colour temperature is lower at longer wavelengths. These observations allow an interpretation in terms of the presence of extended envelopes for these stars and do not differ qualitatively from the observations of WR stars (Kuhi, 1966). There is only a slight trend of these colour temperatures with spectral type. The extent of the envelopes seems to be the real determining parameter. In this respect it should be remembered that Of stars and B-type supergiants with only weak P Cygni-type characteristics show hardly any intrinsic reddening. On the other hand peculiar binaries and symbiotic stars on the average show considerable reddening like the pure P Cygnitype stars. Also, the normal colour indices of $P$ Cygni-type stars are redder with respect to the $B-V$ index and bluer with respect to the $U-B$ index than those in ordinary B-type stars.

\section{Absolute Magnitudes}

Like for the WR stars the determination of absolute magnitudes of P Cygni-type stars is a difficult task. The narrow band photometric systems used by Westerlund (1966) and by Smith (1968) to determine the absolute magnitudes and intrinsic colours of WR stars would be most useful for determining the absolute magnitudes and intrinsic colours of P Cygni-type stars as well. This is especially so since the P Cygni-type stars, with their narrower emission lines, would contaminate even less the photometric system. However, these photometric systems have not yet been used for this purpose and absolute magnitudes and intrinsic colours are based upon $\mathrm{U}, \mathrm{B}, \mathrm{V}$ photometry 
and upon distance estimates from the interstellar $\mathrm{K}$ line. In this respect it should be borne in mind that distance estimates based upon $\mathrm{K}$-line radial velocities and equivalent widths do not always agree nicely since P Cygni-type stars may show stellar components of the $\mathrm{K}$-line, both in absorption and in emission.

Anyhow, from a study of 19 of Beals' pure P Cygni-type stars and $8 \alpha$ Cygni-type supergiants Arkhipova (1964) concludes that the $\alpha$ Cygni-type stars, with a few exceptions, generally are more luminous than most P Cygni-type stars. She distinguishes two classes of P Cygni-type stars: A small group of bright stars with $M_{v} \simeq-7.5$ and a larger group of giants and bright giants of luminosity classes III to Ib and $M_{v}$ between -3 and -5 . On the one hand this may be taken as an illustration of the heterogeneity of the collection of P Cygni-type stars, on the other hand it must be pointed out that a study by Luud (1967) of about the same number of P Cygni-type stars shows that the visual absolute magnitudes are all between -6.5 and -8.5 for spectral types between 06 and A4. From Luud's study one gets the impression that the P Cygni-type stars form as homogenous a group of stars as can be reconciled with their variety in spectral types, all being about one magnitude brighter than the brightest supergiants of their corresponding spectral types. Both Luud and Arkhipova base their conclusions on Beals' distances, but Arkhipova is correcting in a more elaborate way for interstellar absorption. The conclusion at this moment must be that there are a number of bright P Cygni-type stars, about $1^{\mathrm{m}}$ brighter than the B-type supergiants. In Arkhipova's study these are at the same time the stars of earliest spectral type and of highest excitation. Thus, in general the $\mathrm{P}$ Cygni-type stars are a few magnitudes brighter than most of the WR stars; the WR stars of subclasses WN7 and WN8 forming the only exception to this rule.

\section{P Cygni-Type Stars in Binaries}

Very few P Cygni-type stars are known to be members of binaries. Perhaps the best known example is AR Pav, but this is a very complex system (Thackeray, 1959) and very difficult to interpret. A more promising object would be HD 152667. This system has recently been studied by Walker (1971) who finds that the Balmer lines show weak P Cygni-type line profiles of Beals' type III. The mass function of the system is 0.487 which leads to a minimum mass of the system of 4.1 solar masses. Since the light curve shows two unequal minima the total mass of the system cannot be much larger.

Another possible candidate may be HD 98922. Bond (1970) found this star to be a spectroscopic binary, probably with a large velocity amplitude. Spectra taken by the author show that the stronger $\mathrm{H}$ lines show a $\mathrm{P}$ Cygni-type profile superposed on a quite normal B9 absorption. Although no pure P Cygni-type star seems to be involved, this system like HD 152667, is quite interesting and may yield some information about the mass of a star showing the P Cygni phenomenon at least in its $\mathbf{H}$ lines down to H8. A few photometric observations by the author have not yet given a positive answer to the question if the system shows eclipses as well; the observations are being continued. Clearly, the situation with respect to determining masses of 
P Cygni-type stars in a more direct way is not as favourable as for the WR stars of which at least one third is known to be double. Of a large number of bright $O$ and B-type supergiants studied by Hutchings (1970) none, except one, are known to be binaries.

\section{Masses}

Since in the case of the P Cygni-type stars the method of determining masses from binary systems fails because of their nearly complete absence from those systems, the only way of determing masses is through the mass-luminosity relation.

Here we are facing two problems. The first is the uncertainty in the absolute magnitudes. Only for a small number of P Cygni-type stars having some relation to galactic clusters can absolute magnitudes be obtained in another independent way. The other problem is more fundamental: do P Cygni-type stars obey the mass-luminosity relation? The WR stars do not; they are overluminous for their masses. As long as the reason for this discrepancy is not fully understood we can, a priori, say very little about the behaviour of the P Cygni-type stars in this respect. Because of certain similarities to the WR stars (extended expanding atmospheres, emission-line spectra) it may well be that also the P Cygni-type stars are overluminous for their masses.

If, to have some basis of discussion, we adopt the absolute magnitudes as derived by Arkhipova (1964) $\left(M_{v} \simeq-7.5\right.$ for the early B-type stars and between -3 and -5 for later types) the masses of the stars in these two groups as derived from the massluminosity relation are about $100 M_{\odot}$ and between 8 and $20 M_{\odot}$ respectively. If on the other hand, we adopt Luud's (1967c) bolometric absolute magnitudes, the corresponding masses range from 46 to $144 M_{\odot}$ with a mean value at $76 M_{\odot}$. There are strong arguments in favour of these higher masses in the light of the variability and mass loss of the P Cygni-type stars. This will be discussed further in the next section. If, following the suggestion of Arkhipova (1964) the P Cygni-type stars really are to be divided into two groups, the brighter group most probably owes its characteristics to their state of instability against nuclear-energized pulsations (Luud, 1967b; De Groot, 1969). The masses of the stars in the less-bright group are more in the mass range of the WR stars. However, other characteristics are very different so that not too much weight should be attached to this similarity.

\section{Variability}

\subsection{Light VARIATIONS}

P Cygni-type stars normally show light variations. Most of the variations reported have been characterized as 'irregular' and of small amplitude: normally less than a few tenths of a magnitude in at least several days. However, some stars are known to have shown larger variations. P Cygni was discovered in 1600 as a nova and has since varied rather irregularly over a range of more than three magnitudes (Schneller, 1957). In modern times AG Car has shown irregular variations with an amplitude up to two 
magnitudes (Greenstein, 1938; Mayall, 1969). Smaller variations have been reported for HR Car (Hoffleit, 1940; Wisse and Wisse, 1971). When discussing these observations it should be borne in mind that the photometric behaviour of most P Cygni-type stars has been studied quite erratically only, so that possible short-period variations may well have been overlooked.

The only star of which frequent photometric observations have been made is $P$ Cygni itself. Magalashvili and Kharadze (1967a, b) have found that this star, superposed on its slow irregular light variations, shows a very regular variation with a period of 0.500656 and an amplitude of 0.1 . The light curve resembles that of a W UMa system, but this conclusion has been disputed by several authors (Fernie, 1968; Luud, 1969; De Groot, 1969). Also HD 152667 shows small intrinsic light variations superposed on its eclipse variations, but as yet nothing can be said about amplitude and period (Walker, 1971). Apart from light variations due to duplicity quite a number of WR stars have been found to show probably irregular variations of small amplitude (Roberts, 1962; Smith, 1966; Smith, 1967a; Smith, 1967b; Ross, 1961; Kuhi, 1967; Demers and Fernie, 1964). However, different authors do not always agree upon which stars vary and how much (cf. e.g. Ross, 1961, with Demers and Fernie, 1964). This may be largely due to the infrequency of the observations as in the case of the P Cygni-type stars. For the moment we can only conclude that the variations of WR stars like those of P Cygni-type stars are irregular with small amplitude but that short-period variations may exist as well.

\subsection{SPECTRUM VARIATIONS}

In his study of the P Cygni-type stars Beals (1950) has indicated some stars which had shown spectral changes in the first half of this century. However, hardly any stars have been studied in any detail so that we can only rely upon detailed studies of P Cygni itself(Luud, 1966; Luud, 1967a; Luud, 1967b; De Groot, 1969). Luud studied the equivalent widths of most of the stronger lines in the spectrum of P Cygni and also the line profiles of the $\mathrm{H}$ lines. Both authors agree upon the fact that the equivalent widths of all major lines seem to vary irregularly over a range of about 30 per cent. Kupo (1955) and Dolidze (1958) found that in 1951 and 1952 P Cygni showed irregular spectrophotometric variations coincident with the light and colour variations of the star. P Cygni also shows variations in its line profiles (Astafjew, 1967; De Groot, 1969) due to the presence of different shells in the atmosphere. The outermost shell shows radial-velocity variations with a 114-day period.

Other investigations reveal spectral changes in other P Cygni-type stars. In 1952 HR Car had its $\mathrm{HeI}$ lines in emission together with those of $\mathrm{H}, \mathrm{Fe} I$ and [F II] (Henize, 1952) but in 1968 the HeI lines were all in absorption while the rest of the spectrum remained essentially unchanged (Bond and Landolt, 1970). AG Car is a P Cygni-type star surrounded by a low-excitation, ring-shaped shell. P Cygni-type lines in its spectrum were numerous in 1950 (Thackeray, 1950; Thackeray, 1956) but had mostly disappeared in 1968, only remaining present in the stronger Balmer lines (Bond and Landolt, 1970). 
HD 152667, apart from the radial-velocity variations due to its duplicity, shows small intrinsic radial-velocity variations with a period of about 0.6 or less (Walker, 1971) resembling a $\beta$ Cephei-type of curve.

Spectral variations in WR stars are not so firmly established. This may be partly due to the existence of their strong emission lines and their weak continuum which makes the precise measurement of equivalent widths and line profiles very difficult. Obvious intrinsic variations are known in the case of HD 45166 which looked like a WR star in 1922 and was intermediate between Of and WR in 1948 (Anger, 1933; Neubauer and Aller, 1948). Recent work has shown that at least some WR stars show irregular variations of their emission line profiles (cf. e.g. Smith and Kuhi, 1970). They attribute some variations to the fact that lines are formed by selective processes (Underhill, 1968) so that different lines of the same spectrum (e.g. N IV $\lambda 4057$ and $\lambda 3480$ ) are formed in different parts of the atmosphere. Intrinsic variations have also been observed by Bappu (1951).

Underhill (1968) remarks that "it is rather striking how constant WR spectra remain, considering the state of motion believed to exist in their atmosphere". On the other hand, the state of motion believed to exist in the atmospheres of P Cygni-type stars seems to be much more quiet but their variations are more pronounced. Therefore, the spectral variations of P Cygni-type stars probably have a different cause from those in WR stars. Both the light and spectrum variations of P Cygni-type stars probably are closely related to their evolutionary status. Since, however, they form a rather heterogeneous class of objects one should hesitate to draw general conclusions about the class as a whole.

The explanation of the spectral and light variations of $P$ Cygni-type stars is sought in the following facts: From the work of Ledoux (1941) and of Schwarzschild and Härm (1959) it is known that above a certain critical mass a star cannot be stable against nuclear-energized pulsations. Appenzeller (1970) calculated the characteristics of a vibrationally unstable main sequence star of $130 M_{\odot}$ and came to the conclusion that it describes nearly exactly the observations of P Cygni. On the other hand, Stothers and Simon (1968), from a calculation of massive core helium-burning stars with hydrogen-burning shells, find all their models highly stable against nuclear-energized pulsations and propose that any star with a mass less than the critical mass, $\sim 60 M_{\odot}$, on the main sequence should evolve into the blue-supergiant region of the HR diagram without disruption. They believe with Lucy and Solomon (1967) that the mechanism causing the mass loss from the B-type supergiants with P Cygni-type characteristics $\left(10^{-7}\right.$ to $\left.10^{-5} M_{\odot} \mathrm{yr}^{-1}\right)$ is atmospheric radiation pressure. In a later paper Simon and Stothers (1970) compute the evolution of hydrogen burning stars with moderate mass loss whose mass exceeds the critical mass for pulsational stability at the top of the main sequence. They conclude that these models give a good representation of the mass loss of the most luminous group of WR stars. To explain the behaviour of the P Cygni-type stars I prefer Appenzeller's calculations because they account not only for the observed mass loss but also explain nearly all other observed phenomena. The pulsation periods for unstable stars are around 0.5 and 
may explain the 0.5 -period light variations of P Cygni found by Magalashvili and Kharadze (1967a, 1967b). The special variations with a time scale of a few days as found by Astafjew (1967) can be explained in the same way. Since he took only four spectra in one week a more intensive series of observations probably would reveal the shorter period.

The same applies to the 0.5 period radial-velocity variations in HD 152667 found by Walker (1971). He remarks that of the irregular variations in the light curve it is impossible to say whether they are connected with the possible short radial-velocity variations. I would say instead that it is highly probable that upon closer investigation the irregular light variations will reveal a time scale equal to that of the short radialvelocity variations. The only remaining problem in the case of this system is that it probably is not very massive.

\section{Mass Loss}

Combining the radii determined by Luud (1967c) with estimates of particle density from equivalent widths of spectral lines and with measured radial-velocities of outflow (Hutchings, 1970) one arrives at mass-loss figures for the P Cygni-type stars between $10^{-4}$ and $10^{-5} M_{\odot} \mathrm{yr}^{-1}$. An extensive study by Hutchings (1970) has shown that mass losss in $\mathbf{O}$ and B-type supergiants is dependent on both temperature and luminosity; or, in other words, on the UV flux and the surface gravity. The lines connecting stars with the same amount of mass loss follow the zero mass loss evolutionary tracks, suggesting thereby that the rate of mass loss is approximately constant throughout the hydrogen and early helium-burning lifetime of each star. The stars with very high luminosity show mass losses of about $10^{-4} M_{\odot} \mathrm{yr}^{-1}$. For normal supergiants this figure goes down to about $10^{-7} M_{\odot} \mathrm{yr}^{-1}$. Mass loss seems to begin in those stars that have exhausted the hydrogen in their cores. These values of the mass loss for P Cygni-type stars compare with those for WR stars which are of the order of $10^{-5}$ to $10^{-6} M_{\odot} \mathrm{yr}^{-1}$ (Underhill, 1969; Smith, 1966).

For all these stars radiation pressure exerted on the gas in the atmosphere through absorption in resonance lines at wavelengths near the maximum of the stellar-continuum flux is an important driving mechanism for the observed mass loss (Lucy and Solomon, 1970). Besides that there is another circumstance for the brighter P Cygnitype stars. Since they have masses ranging from 50 to $140 M_{\odot}$ many of them are more massive than the critical mass for hydrogen burning main-sequence stars and, therefore, are pulsationally unstable. This may be a very important mass-loss factor. The analogy with the WR stars, where Paczyński, I think, believes that mass loss is due to a similar instability for helium burning stars, is striking.

\section{Evolution}

This last part is only meant as a presentation of some thoughts and facts related to the evolutionary status of the P Cygni-type stars and should not be considered as a well-founded explanation of all observations. 
There are a few examples of the evolution of single objects. Apart from the spectral changes mentioned in Section 8 one might mention HD 190073 which showed a Betype spectrum before 1933 and $\mathrm{P}$ Cygni-type lines superposed on shallow high-rotation lines in 1937-'38. HD 87643 showed only $\mathrm{H}$ in emission in 1909; about 30 yrs later it was reclassified as P Cygni-type; in 1953 Munch listed it as an emission star with WR characteristics (Munch, 1953); finally, in 1968 the spectrum was rather nova-like (Hiltner et al., 1968). Another interesting object is HD 89249 which in 1909 showed only $\mathrm{H} \beta$ in emission, later was reclassified as P Cygni type and in 1968 showed a typical Be-type spectrum. (Hiltner et al., 1968). These are some of the facts concerning the evolution of several individual objects showing the P Cygni phenomenon.

Let me now turn to some thoughts relating certain characteristics of the WR stars with those of P Cygni-type stars. Among the WR stars the WC8 and WC9 stars show the lowest excitation and may therefore, lend themselves best for a comparison with the P Cygni-type stars. The WC8 and WC9 stars have line widths of 10 to $15 \AA$, against more than $30 \AA$ for the other WR stars and less than $4 \AA$ for the P Cygni-type stars. Before this symposium the absolute magnitudes of the WC8 and WC9 stars were still at $-6^{m} \cdot 2$ and nicely intermediate between the other WR stars and the P Cygnitype stars. However, Lindsey Smith has felt obliged to bring this value back to $M_{v}=-4^{m} .8$ for the WC8 stars and $-4^{m} .4$ for the WC9 stars. Since this correction creates some problems with respect to their galactic distribution not all hope for WC8 and WC9 stars to be intermediate between WR stars and P Cygni-type stars need be lost. There is, on the other hand, another problem: If in the P Cygni-type stars the $\mathrm{N}$ overabundance is a real thing, then there may be a case for comparing the WN8 and WN8 stars with them. These WR stars still stand at their high luminosities of $-6^{m} .8$ and $-6^{m} .2$ respectively. Furthermore, these are the WR stars with morepronounced UV absorption edges than the others as Lindsey Smith said yesterday.

Since P Cygni-type stars do not occur in binaries they have not been subject to mass exchange. Furthermore, they show the normal $\mathrm{H} / \mathrm{He}$ abundance ratio. Thus, there does not seem to be any contradiction in concluding that the P Cygni-type stars are burning hydrogen as massive main-sequence stars subject to mass loss through radiation pressure in their resonance lines and/or through the effect of instability against nuclear-energized pulsations. This last effect also explains their short-period light and spectral variations. If anywhere, then Thomas' stars having a corona and an exosphere are to be found among the P Cygni-type stars.

\section{References}

Appenzeller, I.: 1970, Astron. Astrophys. 5, 355.

Arkhipova, V. P.: 1963, Soviet Astron. 7, 51.

Arkhipova, V. P.: 1964, Soviet Astron. 7, 686.

Astafjew, E. R.: 1968, Astrophysics 4, 191.

Bappu, M. K. V.: 1951, Astron. J. 56, 120.

Beals, C. S.: 1940, J. Roy. Astron. Soc. Can. 34, 169.

Beals, C. S.: 1950, Publ. Dominion Astrophys. Obs., Victoria 9, 1.

Bond, H. E.: 1970, Publ. Astron. Soc. Pacific 82, 1065. 
Bond, H. E. and Landolt, A. U.: 1970, Publ. Astron. Soc. Pacific 82, 313.

Caputo, F. and Viotti, R.: 1970, Astron. Astrophys. 7, 266.

De Groot, M.: 1969, Bull. Astron. Inst. Neth. 20, 225.

Demers, S. and Fernie, J. D.: 1964, Publ. Astron. Soc. Pacific 76, 350.

Dolidze, M. V.: 1958, Abastumansk. Astrofiz. Obs. Gora Kanobili Bjull. $23,69$.

Fernie, J. D.: 1968, Observatory 88, 167.

Ghobros, R. A.: 1962, Z. Astrophys. 56, 113.

Greenstein, N. K.: 1938, Harvard Bull., No. 908, 25.

Hiltner, W. A., Stephenson, W. B., and Sanduleak, N.: 1968, Astrophys. Letters 2, 153.

Hoffleit, D.: 1940, Harvard Bull. No. 913, 4.

Hutchings, J. B.: 1968a, Monthly Notices Roy. Astron. Soc. 141, 219.

Hutchings, J. B.: 1968b, Monthly Notices Roy. Astron. Soc. 141, 329.

Hutchings, J. B.: 1969, Monthly Notices Roy. Astron. Soc. 144, 235.

Hutchings, J. B.: 1970, Monthly Notices Roy. Astron. Soc. 147, 161.

Kuhi, L. V.: 1966, Astrophys. J. 143, 753.

Kuhi, L. V.: 1967, Publ. Astron. Soc. Pacific 79, 57.

Kupo, I. D.: 1955, Astr. Cirk. Izdav. Bjuro Astr. Soobshch. Kazan, No. 163, 23.

Ledoux, P.: 1941, Astrophys. J. 94, 537.

Lucy, L. B. and Solomon, P. M.: 1967, Astron. J. 72, 310.

Lucy, L. B. and Solomon, P. M.: 1970, Astrophys. J. 159, 879.

Luud, L. S.: 1967a, Soviet Astron. 11, 211.

Luud, L. S.: 1967a, Astrophysics 3, 172.

Luud, L. S.: 1967c, Izv. Akad. Nauk Est. S.S.S.R. 16, 319.

Magalashvili, N. L. and Kharadze, E. K.: 1967a, Inf. Bull. Var. Stars, No. 210.

Magalashvili, N. L. and Kharadze, E. K.: 1967b, Observatory 87, 295.

Mayall, M. W.: 1969, J. Roy. Astron. Soc. Can. 63, 221.

Morton, D. C.: 1967, Astrophys. J. 150, 535.

Munch, L.: 1953, Bol. Obs. Tonantzintla Tacubaya, No. 8, 27.

Roberts, M. S.: 1962, Astron. J. 67, 79.

Ross, L. W.: 1961, Publ. Astron. Soc. Pacific 73, 354.

Schneller, H.: 1957, Geschichte und Literatur des Lichtwechsels der veränderlichen Sternen, Akademie Verlag, Berlin.

Schwarzschild, M. and Härm, R.: 1959, Astrophys. J. 129, 637.

Simon, N. R. and Stothers, R.: 1970, Astron. Astrophys. 6. 183.

Smith, L. F.: 1966, Narrow-Band Photometry of Wolf-Rayet Stars, Thesis, Australian Natl. Univ., Canberra.

Smith, L. F.: 1968a, Monthly Notices Roy. Astron. Soc. 138, 109.

Smith, L. F.: 1968b, Monthly Notices Roy. Astron. Soc. 140, 409.

Stothers, R. and Simon, N. R.: 1968, Astrophys. J. 152, 233.

Thackeray, A. D.: 1950, Monthly Notices Roy. Astron. Soc. 110, 524.

Thackeray, A. D.: 1956, Vistas in Astronomy 2, 1386.

Thackeray, A. D.: 1959, Monthly Notices Roy. Astron. Soc. 119, 629.

Thomas, R. N.: 1968, in K. G. Gebbie and R. N. Thomas (eds.),Wolf-Rayet Stars, U.S. Government

Printing Office, Washington, p. 1.

Underhill, A. B.: 1968, Ann. Rev. Astron. Astrophys. 6, 39.

Underhill, A. B.: 1969, Astrophys. Space Sci. 3, 109.

Walker, E. N.: 1971, Monthly Notices Roy. Astron. Soc. 152, 333.

Westerlund, B. E.: 1966, Astrophys. J. 145, 724.

Wisse, P. N. J. and Wisse, M.: 1971, Astron. Astrophys. 12, 149.

\section{DISCUSSION}

Thomas: Could you sketch how Ca II looks?

De Groot: The normal interstellar components (Figure 1) lie at -10 and $-23 \mathrm{~km} \mathrm{~s}^{-1}$; they are quite strong. Sometimes, on the shortward flank of the component at $-10 \mathrm{~km} \mathrm{~s}^{-1}$, another can be seen at $-16 \mathrm{~km} \mathrm{~s}^{-1}$. Then there are two much weaker components at +17 and $+26 \mathrm{~km} \mathrm{~s}^{-1}$. Finally there are the two weak components at -36 and $-49 \mathrm{~km} \mathrm{~s}^{-1}$. 


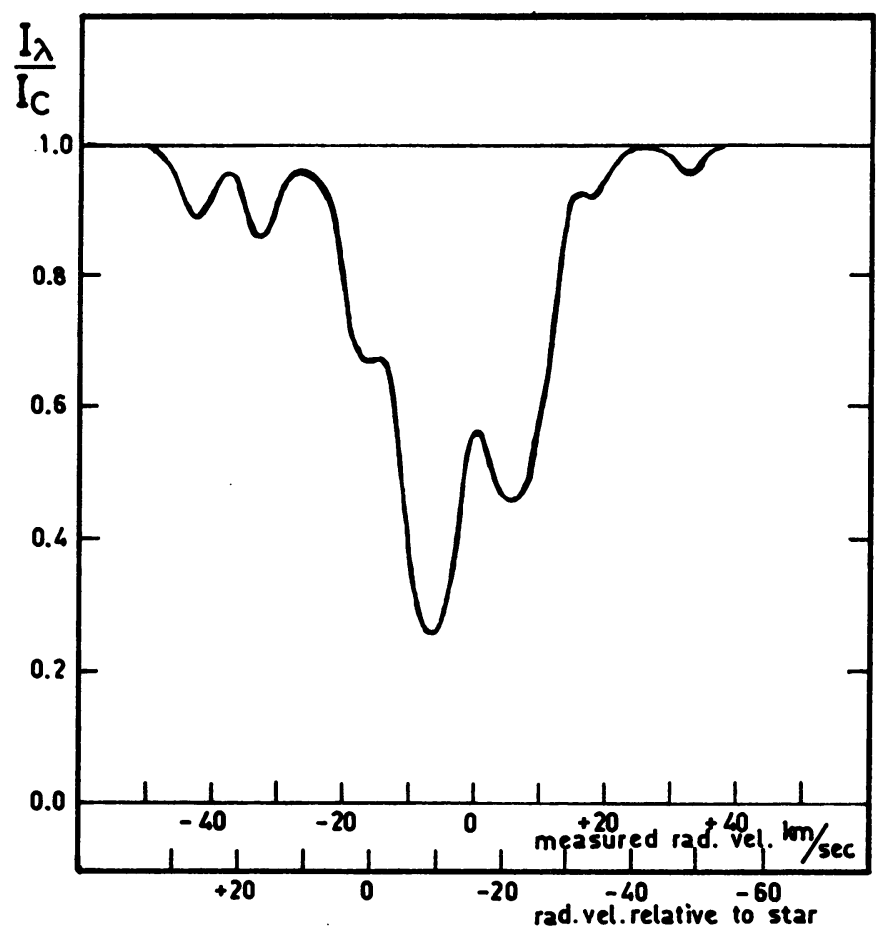

Fig. 1.

Morton: Which ones are permanent and which ones appear sporadically?

De Groot: The components at -10 and $-23 \mathrm{~km} \mathrm{~s}^{-1}$ are always there. The components at $0,+17$ and $+26 \mathrm{~km} \mathrm{~s}^{-1}$ are quite permanent, that means visible roughly on one plate out of three. The components at -36 and at $-49 \mathrm{~km} \mathrm{~s}^{-1}$ I have seen only once.

Van Blerkom: Do you see the same sort of behaviour with the sodium lines?

De Groot: That I did not check yet. The one at $-36 \mathrm{~km} \mathrm{~s}^{-1}$ is sometimes showing up in the $\mathrm{Na}$ I D lines as well, but not at the corresponding positive velocity. However, at this wavelength the number of plates is only 5 .

Kuhi: I am just wondering how you can so definitely rule out the interstellar component?

De Groot: That was based on the fact that when I found these components, I was more or less convinced that they were really there, and then the fact they just fit was such a nice coincidence that I wanted to explain it in this way.

Paczyński: I still do not understand what the coincidence could possibly mean.

De Groot: The mean velocity of two particular components is the stellar velocity. It means that you have a stream going out and one coming back at the same velocity with respect to the star.

Paczyniski: So you have matter ejected from the star, and at the same time other matter falling back. I am trying to visualize the picture. It seems difficult.

Thomas: No, if you are willing to accept an outward decelerated field, you can accelerate it for a while and then decelerate it for a while and then throw it back. I am not suggesting anything.

Paczyński: You have two components always present. So you have a more or less stationary situation.

Thomas: It depends on where the excitation is. If, for example, I throw it out, its excitation changes and if it comes back in again its excitation changes. You pick the locus of where $\mathrm{Ca}$ is predominantly $\mathrm{Ca}$ II. It is a way to make a model.

Underhill: $I$ think it is interesting that these inward components are seen against the emission because that emission gives you a background against which you see some absorption. After all, in stars like 
31 Cygni you see clouds going in and out, at the same time, when you are looking at it. You only have the $B$ star at one side of the $K$ star but you can see plus and minus components at the same time. It is a satisfactory description.

Kuhi: Could I also ask whether you have looked at any other $O$ and B stars in the vicinity, to see what the interstellar component out there looks like.

De Groot: I remember from the work of Adams that there is another star very close to P Cygni. It has the same four components at the same velocities.

Bappu: But one finds a temporal change. That is the striking feature. It is not a permanent feature. One of those components comes and goes.

De Groot: It has happened only once. I did not see all of them always, but quite often. The real interstellar ones are always there.

Thomas: So when you average the -49 and the +17 , which you call interstellar, what you are seeing is really a shell associated with the star. How many shells associated are interstellar?

De Groot: No, it is what you would call a circumstellar line.

Thomas: So this is something which is partly going out and partly coming back. Is that what you suggest?

De Groot: I suggest, and it seems to be quite controversial, that you see here the sum of the interaction between the expanding envelope and the interstellar medium.

Paczyński: Is there any observational evidence that could disprove the suggestion that P Cygni behaves, as far as the light curve is concerned, like a W UMa system with a period of half a day and an amplitude of $0.1 \mathrm{mag}$.

De Groot: Fernie calculates this case. From the mass-luminosity relation, he finds a mass of about one hundred solar masses. If you take one particle and let it go round this star which has a large radius as well, then the velocity is something like $10^{4}$ kilometers per second, and that is not possible.

Paczyniski: I have asked you about the observations. According to the results of Appenzeller, a main sequence star of about 100 solar masses may become vibrationally unstable and may increase its radius by a factor of four, and decrease its effective temperature by a factor of two, and look like $P$ Cygni itself. The pulsation period for the main sequence star is about 10 hours when the star expands by a factor of four. Therefore, there is no theoretical reason at present to deny a half-day period for the luminosity variation of $P$ Cygni.

De Groot: I think there are photoelectric observations of Alexander and Wallerstein (Publ. Astron. Soc. Pacific 79, 500, 1967), who have tried to check the half-day period, and have found no variation. I would not reject at first the half-day period, but reject at least the W Ursae Majoris system idea, and explain any variations as pulsational instability.

Paczyniski: That is why I was asking for the observational evidence.

De Groot: I think the observations pointing to the half-day periods are still better than the ones denying it, since they are more numerous, but this is still a controversial point. May be we can summarize by saying that normally the variations are quite irregular.

Thomas: Could you tell us what density and radii and velocity you use, that yield a mass loss of $10^{-4}$ to $10^{-5} M_{\odot}$ per year?

De Groot: For stars between 46 and 144 solar masses you have radii between 40 and 80 solar radii; roughly 100 solar masses give a radius of 60 solar radii.

Paczynski: What do you mean by a mass-radius relation?

De Groot: The luminosity-radius relation.

Paczyński: Luminosity-temperature-radius?

De Groot: Yes, the luminosity-temperature-radius. You have the logarithm of the electron densities which range between 12.5 to 13 , and you have velocities of one hundred to two hundred kilometers per second. Now if you combine these you come to mass-loss figures in this range. The only thing is that you have to believe this. Hutchings has more extensively studied mass loss in OB supergiants and he finds that it is dependent on both temperature and luminosity. He draws a HR diagram; you have the main sequence, you have the evolution of a thirty and a fifteen solar mass star and the stars showing the highest degree of mass loss are all in the $30 M_{\odot}$ region and above; we should go down to find lower masses in order to the stars of less and less mass loss.

Thomas: And what happens if you go in the other direction?

De Groot: You have more mass loss. This is only for supergiants. In OB supergiants you have mass losses of $10^{-5}$ to $10^{-7} M_{\odot}$ per year, so a little bit less, and the lines of constant mass loss are more or 
less parallel to the lines of the evolution off the main sequence of the stars of the masses of the OB supergiants.

Thomas: And how do I infer the mass loss of the OB supergiants?

De Groot: More or less as it has been done for the P Cygni stars.

Thomas: For P Cygni I have some kind of a number to give me a number.

De Groot: Because you have in the $\mathrm{OB}$ supergiants nearly always a velocity at $\mathrm{H} \alpha$ from the $\mathrm{P}$ Cygnitype profile, and you can take your velocity from there. Then, you have the observations in the rocket UV.

Paczyński: The rocket UV observations as interpreted by Lucy and Solomon indicated the mass loss rates of $10^{-8}$ solar masses per year. This is considerably less than the rates you quoted.

Morton: We must not forget the uncertainties in both the theoretical and observed rates of mass loss. For the theoretical estimate Lucy and Solomon put in one mechanism and found about $10^{-8}$ solar masses per year. This number can be taken as a lower limit, but the observations show that many resonance lines are effective rather than a single dominant line at each temperature predicted by the calculations. On the other hand, the higher rates derived from the spectra often depend on large, poorly established corrections for unseen ions.

Thomas: What makes you think that it will disagree? What is the evolutionary time-scale for these kinds of solar masses?

Paczyniski: It is less than $10^{7}$ years on the main sequence and less than $10^{6}$ years in the supergiant phase.

De Groot: It is less interesting if it concerns a mass that comes from a mass of 100 solar masses, because then it may be only one per cent anyhow.

Conti: Theoretically we do not know. It may make a whole lot of difference.

De Groot: You could ask why we do not see surrounding P Cygni stars, anything like shells or rings or nebulae. We have to conclude that the extended atmospheres and the matter which is lost is so dense that the region cannot be very large in extent and so it escapes discovery. If you build a Strömgren sphere and you fill it and you make it very dense, the ionizing radiation cannot go very far from the star, so the nebulae you will detect are to be small ones.

Thomas: You say this is so dense that I cannot see it?

De Groot: No, the matter which leaves the star is so dense that the ionizing radiation from the star does not go very far.

Thomas: But if I take the implicit suggestion of velocities that can be a thousand kilometers per second, then you will see them in the rocket UV. I think the absence of a shell is very embarrassing.

Morton: You have the same problem with Wolf-Rayet stars.

Thomas: Oh, sure. But you still have an embarrassment. Would you think that in terms of the observations that if they are there, you should see them? Or would you accept what Anne Underhill said, that may be we have not looked enough?

De Groot: I think we did not look at enough areas. In the southern hemisphere, Thackeray has found very few, two or three objects which have real P Cygni characteristics and are associated with visible nebulae.

Thomas: Oh, he does find some!

De Groot: Yes. I did not say there were none. But the number must be impressively low.

Thomas: Do they have any particular characteristics; are they different from other kinds of shells?

De Groot: I think that most of them are circular. The star is more or less in the center.

Paczyniski: Are the central stars hotter than average P Cygni stars?

De Groot: Most of Thackeray's were B's. They were not excessively hot stars.

Paczyniski: The late B's could hardly ionize the nebulae.

De Groot: I think they were probably early B's, but I should have to look it up. I do not remember.

Smith: I think the WN7 and WN8 spectra are more like the P Cygni spectra than are the WC spectra.

De Groot: Yes.

Smith: The WN7's and WN8's are still estimated to have average absolute visual magnitudes -6.8 and -6.2 respectively. I think the WN's are the most closely related to the P Cygni's, in visual appearance.

De Groot: If you talk about line widths and about excitation, then, the answer is no, but if you take some other characteristics, especially the fact that $\mathbf{N}$ may be a bit overabundant in P Cygni, because at least the $\mathrm{N}$ lines are very marked, then you come to WN7 and WN8. The P Cygni stars 
do not occur in binaries, apart may be from a very few; they cannot have been subjected to mass exchange and, therefore, at the moment I do not have the problem to assume that they are really helium-burning stars at later phases of their main-sequence life and show this pulsational instability. If I had to classify the phenomena on the scheme of Thomas, I would say that they have a corona and an exosphere. Regarding mass and momentum transfer, etc., that is very difficult to say.

Morton: What is the mass of a typical P Cygni star? Do the P Cygni stars have the problem of the Wolf-Rayet star in the sense that the mass is much less than we would estimate from the mass-luminosity relation?

De Groot: At the moment it is not clear where we stand on that. But to summarize, the one bit of evidence was one star with a mass not more than 4 solar masses, that somebody just mentioned.

Walborn: It was HD 152667. This star is a BO supergiant in Sco OBI, and it has an absolute magnitude of -6.7 , which is consistent with other stars with similar spectra in the region. The mass derived, according to that analysis, is mot nuch greater than $4.1 M_{\odot}$.

Morton: It just may be that the P Cygni stars have the same problem that the Wolf-Rayet stars have, that they are very over-luminous for their masses.

De Groot: Apart from the fact that you then do not really know their masses, you would introduce the problem that you do not understand the cause of the difference either. You have to invent something else.

Paczyński: If you feel attached to the vibrational instability of massive stars you should favour lower masses. If these stars are overluminous indeed, it means that their hydrogen content must be low. The critical mass for the vibrational instability decreases with the hydrogen content. At the limit of zero hydrogen, the critical mass is $\mathbf{1 5}$ solar masses. It means that you may reduce this critical mass very considerably. If there is indeed anomalous abundance of $\mathbf{N}$, you may speculate along the lines you suggested yesterday: these stars may be mixed. Of course, this is just a speculation.

Underhill: What is the period of HD 152667 ?

De Groot: 7 days point something.

Underhill: I was wondering whether you are looking for eclipses which are not known. Is it possible in this star that the apparent supergiant character of it could be partly due to gas streams in a reasonably close pair. Is it double-lined, do you know?

De Groot: No, it is single-lined, so it gives the mass function. The star which shows the P Cygnitype line profile is the star which is in front during eclipse.

Underhill: Does it have a good light curve?

De Groot: Yes; The light curve has not been established very completely, but at least one can say that there are eclipses of 0.10 or 0.15 mag.

Kuhi: I have several questions. One is in connection with the color temperatures that you quoted earlier. What wavelengths did those refer to?

De Groot: The results I mentioned refer to the region between 3500 and $6000 \AA$ and in several stars there is a trend of the colour temperature decreasing with increasing wavelength.

Kuhi: I raised a question about that very same point, that in OB supergiants, as well as in WolfRayet stars, you do find that the colour temperature that you get is definitely a decreasing function of increasing wavelength. So it is not clear what the colour temperature means at all. And then I have another question. I am more intrigued by the presence of the circumstellar $\mathrm{Ca}$ II lines. Is there any way that you can even guess the size of the distance from the star at which these occur, or can you speculate on it?

De Groot: The lowest excitation stellar lines, that is the Balmer lines and, I think, Mg II, are formed at $2 \frac{1}{2}$ stellar radii. That means that at $2 \frac{1}{2}$ stellar radii we have the amount of excitation that produces the hydrogen lines. This would be at about $160 R_{\odot}$. This means that you have to go a little bit farther out to find the excitation region for the calcium lines. I cannot say how much farther out but it gives you an idea; it should be between 3 and 10 stellar radii.

Paczyniski: Can we observe the component of the $\mathrm{Ca}$ line that could show a large expansion velocity? In other words, if you extrapolate your picture going out very far from the star, you are going into steadily less excited regions of the envelope, you may approach the region where the $\mathrm{Ca}$ line should be visible? Is there anything at a few hundred $\mathrm{km} \mathrm{sec}^{-1}$ ?

De Groot: I could not say at the moment. I have a list of identifications, and at two angstroms shorter there is an unidentified weak absorption line. But it has connected with it an unidentified weak emission line, which should not be there. It has been seen only in one spectrogram, so I would hesitate to identify that as a stellar $\mathrm{Ca}$ II absorption line. 
Morton: I can understand the $\mathrm{Ca}$ II resonance lines originating in some unusual clouds but I would be surprised if these lines are significant in most of the expanding shell. Close to the star the ion state is certainly going to be primarily $\mathrm{Ca}$ III, and to a first approximation the ionization stays constant as the material flows out. The decrease in the radiation flux is compensated by the decreasing electron density.

Paczynski: You mean it has to be really far away, in the interstellar medium.

Morton: I could imagine you might have some very particular cloud or prominence as in the Sun, where the material goes up and comes down again.

Underhill: That is about what I was just thinking. The point is, you see multiple components, atleast one up and one down. Perhaps you are seeing small condensations. The only way to bring your degree of general excitation down is to increase the pressure, and may be it just happens that this time you saw some small irregularities going out in the density. There is no real evidence one way or the other in the P Cygni stars that it is an absolutely uniform expansion. The types of observation we have in hand, can show that you have to have an eclipsing star to see that. And for that reason, this HD 152667, because it does show an eclipse, would be rather useful to be observed at rather high resolution, particularly when one star goes behind the other in order to see if you have any evidence for an irregular flow.

Paczynski: One more comment. The escape velocity from those masses and radii which are given in the table is about $600 \mathrm{~km} \mathrm{~s}^{-1}$. This is considerably more than what is observed. So in fact there is no clear observational evidence for rapid mass loss.

Morton: I am willing to bet that the UV spectra will show material moving outward faster than the escape velocity, just by analogy with the $O B$ supergiants where we find $200 \mathrm{~km} \mathrm{~s}^{-1}$ in the visible and $2000 \mathrm{~km} \mathrm{~s}^{-1}$ in the UV.

Thomas: But you do not know how much of this material is lost. These observations are all based on essentially photospheric radii. In the rocket UV this can really be extended to almost anything. So I can have a small fraction of the matter at escape velocity and a large fraction not at escape velocity.

Morton: The resonance lines in the far-ultraviolet spectra should show where the majority of the matter in the shell is.

Thomas: The majority of the matter excited to that level.

Morton: But in the far ultraviolet we can observe the absorptions from the ground state.

Thomas: Of what lines?

Morton: Almost all the abundant ions.

Thomas: Of $\mathrm{Ca}$ and $\mathrm{C} \mathrm{Iv}$ ?

Morton: $\mathrm{C}$ IV is observable but not C III since its resonance lines lies shortward of the Lyman limit.

Thomas: So that is four resonance lines in that region of the atmosphere where I excite C IV.

Morton: But you also can get $\mathrm{C} \mathrm{III}$ and $\mathrm{C}$ II.

Thomas: So if I only see C IV, that does not tell me how much the mass density is. It can be very much larger.

Morton: You must apply an ionization correction based on observations of $\mathrm{C}_{\mathrm{III}}$ and possibly $\mathrm{C}$ II resonance lines.

Thomas: So we are very uncertain until we observe these. 1 am sure you are right on all the possibilities you have specified. I just wish I know what they were.

Wood: This is another topic, but I wonder if the measurement of expansion velocities from a plate of P Cygni is not related to the question of the velocity variation with distance out from the photosphere of the star?

Are you not making a systematic error if you measured both emission edges or the redward edge of the emission? One never sees the maximum velocity of the expansion of the back side of the star. You do see the front side in absorption. And does the error not depend on the geometrical dilution factor, that is, how big the shell is with respect to the photosphere?

De Groot: There are several geometric factors which determine the shape of the profile, and they determine the relation in density between the emission and absorption line profiles. Normally you will have an overlap between the absorption and emission lines. And you would expect that you measure a higher-velocity absorption line for the stronger emission, just because the short part of the absorption line has been filled in. This has been corrected for as good as I could at that time. You can determine this relation from lines with various emission intensities, and then apply a correction, and then come out, at least for the hydrogen lines, with nearly the same velocities for all the Balmer lines. And then, from the densities, you can show from the Balmer decrement that they are all optically 
thick, that they are all formed in the same region, so they should have more or less the same velocities. Then you are more or less assured that what you did was all right.

Wood: Was it an iterative process, then? You first measure the expansion and some line strengths, calculate the geometry, then go back and correct the velocity? Is that it?

De Groot: No, I estimated the influence of the emission lines with different strengths. The other part of the reasoning is that you can plot the intensities of the hydrogen lines against the Balmer number, and then you find that they are all optically thick. Then you say they should all be formed in more or less the same part of the atmosphere and over a very large range of depth. Then, they should have the same velocity, and with your correction for emission you can have more or less the same velocity and be somewhat consistent and say the correction was probably quite right.

Wood: You do not then measure the highest point in the emission against the lowest point in the absorption?

Underhill: That is the idea you start with, I think, Then you say, with a very weak emission next to a big, strong absorption, it is probably not much distorted. You work your way out to the stronger lines. Then you start reflecting profiles, trying to get a consistent fit. It is explained in De Groot's thesis.

Kuhi: I have two comments: One, to throw the situation into complete confusion, HD 190073 has a fantastically peculiar spectrum, and I am sure you know. The $\mathrm{Ca}$ II $\mathrm{H}$ and $\mathrm{K}$ lines are in emission in that star, and have two, or is it three (?) violet displaced absorption component, indicating a huge amount of material streaming out in the ionized calcium resonance lines.

De Groot: You find emission components for the $\mathrm{Na}$ I $\mathrm{D}$ lines in P Cygni. They are not very strong, but they are definitely there. This is only a question of a shell or an envelope which is denser or less dense.

Kuhi: I am not objecting to anything you are saying, I am just trying to connect it to the circumstellar $\mathrm{Ca}$ II lines. The other thing I wanted to mention is that nobody here so far has mentioned anything about the infrared observations of the P Cygni stars. The thing is that many of them, if not all, (the observations are very spotty), are very bright in the infrared in the sense of having excess radiation at 5,10 and $20 \mu$. No matter how you look at it, this suggests an awful lot of material around the star, may be in the form of dust, may be not. The appearance in the infrared is sometimes accounted for by the presence of free-free emission, and in other cases there is a very distinct bump, which looks like a $10 \mu$ dust bump. It is all connected with material leaving the star, and in a group of a dozen or so stars surveyed spectroscopically as well, the excess seems to be positively correlated with the rate of mass loss.

Morton: Do the Wolf-Rayet stars have a similar effect or not?

Kuhi: They have been looked at, but unfortunately aside from a few, they are all a little bit on the faint side for present equipment. Consequently, nothing has been found: certainly no very large excess exists since it would have been detected easily in the WR stars which have been looked at.

Morton: What about the Of stars?

Kuhi: Some Of stars have been attempted and they do show a slight infrared excess.

Morton: What about ordinary B supergiants like $\varepsilon$ Orionis?

Kuhi: I do not think they have been looked at. The list of things people have been observing in the infrared is a very spotty one. They just pick things that look as if they will be good candidates, i.e., have emission lines. No systematic study has been done, but I think that any star that is throwing out material as evidenced by P Cygni profiles or broad emission lines will probably have an infrared excess.

Underhill: An interesting question is how much is free-free and how much is due to a dust belt. Now with the shell stars, for instance, it turned out that a good deal of it is free-free. The intensity seems to be correlated with the intensity of $\mathrm{Fe}$ II emission lines. That makes sense to a spectroscopist, but if you look a little bit further in the literature they were bubbling over themselves with having rings of dust forming suddenly outside Be stars!

Kuki: We do not know. That is why I said there were two groups: The ones that have a smooth increase in excess which will go out to some 10 or $20 \mu$, and these may be free-free, but there are many others that are not.

Underhill: How big are the observed rings? I think there are one or two. But it is very interesting. How do they get rings out there?

Walborn: Just one comment I wanted to make. In talking about the P Cygni stars, we have been implying they are a rather homogeneous group with regard to luminosity, although you indicated 
quite a temperature range. I seem to recall from Beals' old paper that he found this type of phenomenon in a wide range of objects, not only a wide range of spectral type, but also in several that he thought were of quite low luminosity, so I would like to suggest that perhaps there is a 'P Cygni phenomenon' also, which may occur in different types of objects, and we have been considering only one category. More specifically, I wanted to say that I think even among the high-luminosity group the term 'P Cygni stars' is a little inhomogeneous. On the one hand, we have objects like P Cygni itself which we know had an outburst and has almost a complete P Cygni spectrum, nearly all of the lines having $P$ Cygni profiles; on the other hand, we have very-high-luminosity supergiants which, as you indicated, have a P Cygni profile at $\mathrm{H} \alpha$ only, and also it seems to be a phenomenon of varying degrees in that in many of them you see it at $\mathrm{H} \beta$ also, that is, the only $\mathbf{P}$ Cygni profile in the classification region is at $\mathrm{H} \beta$. HD 190603 is an example of this. And finally, there are the two Of stars associated with NGC 6231, which, as pointed out specifically by Schild, Hiltner and Sanduleak (Astrophys. J. 156, 609, 1969), have very similar spectral types, very similar absolute magnitudes ( -7.4 and -7.1 , yet only one of them has a complete $P$ Cygni spectrum, very similar to $P$ Cygni itself although it is an O8f star. The other one is actually $0{ }^{m} 3$ brighter in absolute magnitude and it has a $\mathbf{P}$ Cygni profile only at $\mathbf{H} \boldsymbol{\beta}$. So these are two very similar yet very different stars.

Underhill: If you look at $\mathrm{H} \alpha$ from a BIa supergiant, you may get a reversed P Cygni emission. I have observed it several times in high dispersion spectrograms.

Morton: What do you mean by 'reversed' P Cygni emission?

Underhill: A profile consisting of a weak emission hump, usually less than $5 \%$ on the violet side and the absorption definitely to the red of where you think your line should be.

Thomas: What kind of star do you see this in?

Underhill: B supergiants. $\eta$ Canis Majoris had it in some plates De Groot took for me in 1970. Thomas: So we have an implosion phenomenon.

Underhill: $\chi^{2}$ Orionis has had it. I mention this to say that the combination of emission and absorption around $\mathrm{H} \alpha$ in the BIa stars does not clearly indicate a simple expanding atmosphere. It is quite variable. These things change. With 55 Cygni, we have gone through a whole series of observations, and sometimes there is no absorption, sometimes it is here, sometimes it is there. Sometimes it is a little faint hump of emission and sometimes nothing.

Walborn: Struve has notes about $\beta$ Ori and $67 \mathrm{Oph}$, too, in which he reports variable emission in the hydrogen lines. 